Article

\title{
A Novel Neural Network-Based Technique for Smart Gas Sensors Operating in a Dynamic Environment
}

\author{
Hakim Baha * and Zohir Dibi
}

Laboratoire d'Electronique Avancée, Département d'Electronique, Université de Batna, 05 Avenue Chahid Boukhlouf 05000 Batna, Algeria; E-Mail: zohirdibi@yahoo.fr

* Author to whom correspondence should be addressed; E-Mail: hakim_bahha@yahoo.fr; Tel.: +213 3380 5494; Fax: +21333805494.

Received: 9 September 2009; in revised form: 27 October 2009 / Accepted: 30 October 2009 / Published: 11 November 2009

\begin{abstract}
Thanks to their high sensitivity and low-cost, metal oxide gas sensors (MOX) are widely used in gas detection, although they present well-known problems (lack of selectivity and environmental effects...). We present in this paper a novel neural network- based technique to remedy these problems. The idea is to create intelligent models; the first one, called corrector, can automatically linearize a sensor's response characteristics and eliminate its dependency on the environmental parameters. The corrector's responses are processed with the second intelligent model which has the role of discriminating exactly the detected gas (nature and concentration). The gas sensors used are industrial resistive kind (TGS8xx, by Figaro Engineering). The MATLAB environment is used during the design phase and optimization. The sensor models, the corrector, and the selective model were implemented and tested in the PSPICE simulator. The sensor model accurately expresses the nonlinear character of the response and the dependence on temperature and relative humidity in addition to their gas nature dependency. The corrector linearizes and compensates the sensor's responses. The method discriminates qualitatively and quantitatively between seven gases. The advantage of the method is that it uses a small representative database so we can easily implement the model in an electrical simulator. This method can be extended to other sensors.
\end{abstract}

Keywords: gas sensor; ANN; implementation; ABM; corrector 


\section{Introduction}

ANNs (artificial neural networks) are used in instrumentation to model complex systems because of their multivariable capability and strong non-linearity. With ANNs the extrapolation errors both inside and outside the calibration range are lower [1]. ANNs are very efficient for dynamic matter problems, offering the advantages of simple implementation and less computation demands, compared to numerical models [2].

Metal oxide sensors (MOX) are one of the most popular technological choices for sensor arrays, due to their high sensitivity [3]. Their main disadvantage is a lack of selectivity. The working principle of these sensors is based on the variation of their conductivity in the presence of oxidizing and reducing gases. The magnitude of the response depends on the nature and concentration of the gas, and on the type of metal oxide [4]. Researchers have examined some aspects of the non linearity of the MOX response to certain gases [5-7], like its dependence on the temperature [8,9], but the dependence on relative humidity and gas nature was not studied and no model of the MOX sensor has been implemented in a simulator.

The subject of linearization and compensation has been considered in different forms and stages; some cases studied include the use of analog to digital converters [10,11], while other work has focused on improving the nonlinear response of specific sensors, like the thermistor [12] and the Hall effect current sensors [13]. Numerical methods have been developed using modern technologies capable of computing linearization algorithms [14,15]. ROM memories are also used to save data tables and to solve the linearization problem [16]. They provide a limited solution to the complex problem under the assumptions that the range of variation of environmental parameters is small and that the influence of the environmental parameters on the sensor characteristics is linear.

To enhance the selectivity of metal oxide gas sensors various strategies have been used by different research groups, including the improvement of the sensitive material [17,18], the use of sensor arrays together with pattern recognition techniques [19,20], and measurements performed in dynamic operation mode [21,22]. In a dynamic environment these strategies don't take the influence of the environmental parameters into consideration or they provide a limited solution to the problem under the assumption that the range of variation is small and that influence on the sensor characteristics is linear.

Figure 1. Multisensory system used.




For those purposes, using artificial neural networks we have designed a model and a corrector for each one of three industrial MOXs (TGS822, TGS821 and TGS813, by Figaro Engineering) and a selective module with the aim of improving the selectivity and eliminating the environmental effects. The diagram of the method used is presented in Figure 1. The MATLAB interface was used during the design phase and optimization; the results (optimal architecture, bias and weights of the network) are used for the implementation of the model, the correctors and the selective module as components in the PSPICE simulator library.

\section{Sensor Characteristics}

We illustrate the design of the TGS822 gas sensor model and the same procedure was followed for the TGS821 and the TGS813 units. According to experimental results [23], the TGS 822 sensor used to detect the gas concentration has a nonlinear sensitivity (Figure 2a; representation is in logarithmic scale) and is dependent on the temperature and humidity of the environment (Figure $2 b$ ) where it is placed. $R_{0}$ is the sensor's resistance in 300 ppm of ethanol, while $R_{S}$ the sensor's resistance to different concentrations of various gases.

Figure 2. (a) TGS822 sensor's sensitivity feature [23]. (b) TGS822 dependence on temperature and relative humidity [23].

(a)

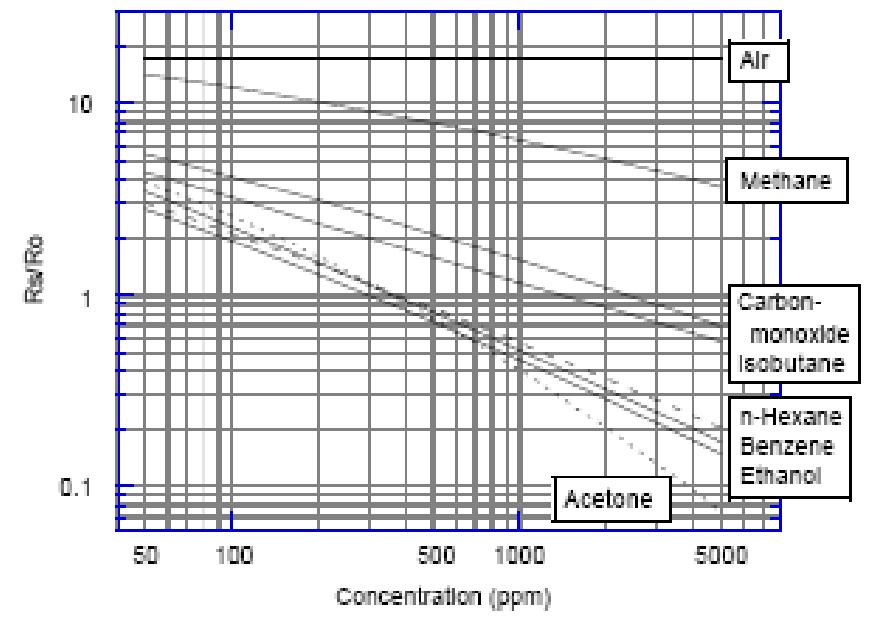

(b)

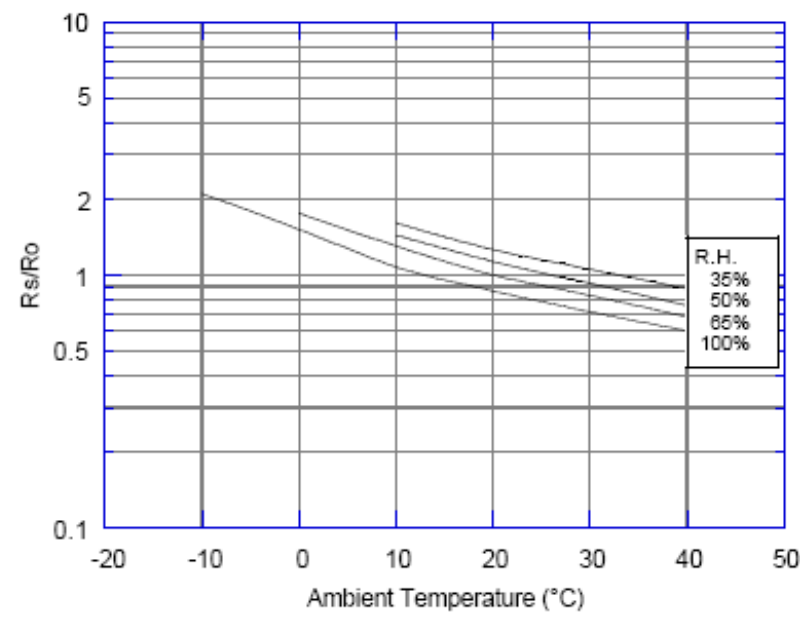

\subsection{The sensor's use}

In Figure 3 a basic gas concentration measurement circuit with the TGS822 sensor is presented. The variation of the TGS sensor's resistance is indirectly measured by the voltage drop appearing on the reading resistance $\mathrm{R}_{\mathrm{L}}$. If a gas like methane, butane, propane etc. comes into contact with the sensor's surface, it's resistance reduces in correlation with the gas concentration present. To work with the features of the sensor provided, in this case, by Figaro, we have to process this output voltage $\left(\mathrm{V}_{\mathrm{RL}}\right)$ and achieve the sensor's resistance $\mathrm{R}_{\mathrm{S}}$ with Equation 1:

$$
\mathrm{R}_{\mathrm{S}}=\left(\frac{\mathrm{V}_{\mathrm{C}}}{\mathrm{V}_{\mathrm{RL}}}-1\right) R_{L}
$$


Figure 3. Measurement circuit with TGS sensor.

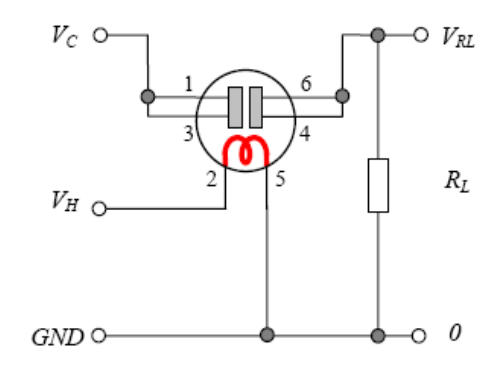

The configuration of the sensor's connected circuits has to ensure the following conditions:

- $\mathrm{V}_{\mathrm{C}}$ can be $5,6,12$ or $24 \mathrm{~V}$.

- $\mathrm{V}_{\mathrm{H}}$ heating voltage has to be $5 \mathrm{~V} \pm 0.2 \mathrm{~V}$.

- The power supply on the sensor maximum $15 \mathrm{~mW}$.

If we determine the sensor's sensitivity feature under standard testing conditions, this characteristic has to coincide with the sensitivity feature, provided by the manufacturer, because this feature is determined with a relative representation of the sensor's resistance. Standard testing conditions, indicated by Figaro are:

- Atmospheric conditions: temperature $20 \mathrm{C} \pm 2{ }^{\circ} \mathrm{C}$ and relative humidity $65 \% \pm 5 \%$

- $\mathrm{V}_{\mathrm{C}}: 10 \pm 0.1 \mathrm{~V}, \mathrm{~V}_{\mathrm{H}}: 5 \pm 0.05 \mathrm{~V}, \mathrm{R}_{\mathrm{L}}: 10 \mathrm{~K} \pm 1 \%$

- Time for the sensor's supply maintenance: seven days or more

- Testing gas: ethanol

\subsection{Sensor parameters}

- Heating resistance: $38.0 \Omega \pm 3 \Omega$

- Sensor's resistance: $1 \sim 10 \mathrm{~K} \Omega$ at 300 ppm ethanol

- Resistance ratio:

$$
\frac{R_{S} \text { in Ethanol } 300 \mathrm{ppm}}{R_{S} \text { in Ethanol } 50 \mathrm{ppm}}=0.4 \pm 0.10
$$

Once the value $\mathrm{R}_{0}$ is measured, the sensor's resistance at different concentrations of various gases and different temperature and relative humidity will be determined as follows: with relation (1) we calculate $R_{S}$, then from Figure $2 b$, for temperature and relative humidity, we read the value $R_{S} / R_{0}$ under the given conditions and we obtain a value $x$. We multiply the value $x$ by $\mathrm{R}_{0}$ and we obtain a value $y$. We divide $\mathrm{R}_{S}$ by $y$ and we get $\mathrm{R}_{S} / \mathrm{R}_{0}$, uninfluenced by temperature and relative humidity, with whose help, from Figure 2a, we will determine the appropriate concentration.

\section{Neural Network Model}

Using the MATLAB interface and based on experimental results from [23], according to the previous paragraph a database is created and arranged as $(\mathrm{S}, \mathrm{T}, \mathrm{RH}, \mathrm{C})$ input and $\left(\mathrm{R}_{\mathrm{S}}\right)$ as output where: 
S: Selecting the gas

$\mathrm{T}$ : Absolute temperature

RH: Relative humidity

C: Gas concentration

$\mathrm{R}_{\mathrm{S}}$ : Sensor resistance

We suppose that Ro $=10 \mathrm{k} \Omega$

Most of this database is used mainly in the training phase by using the LP algorithm (back propagation of error), the remaining data are used to test and validate the model. The diagram in Figure 4 illustrates the direct modeling of the sensor, where:

Yd: Desired output

Y: Network output

e: Modeling error

Figure 4. Modeling of the TGS822.

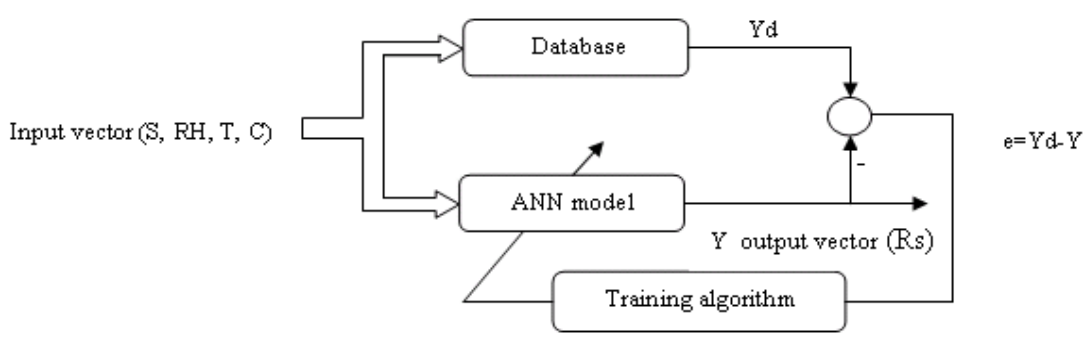

To optimize the model architecture an iteration algorithm is used which consists in evaluation of the total error as a function of layer number and the number of neurons per layer and after many tests of different ANN models. The architecture optimized and that produces the smallest error is summarized in Table 1.

Table 1. Summary the model's optimized parameters.

\begin{tabular}{lll}
\hline Property & Characteristic & \\
\hline Database & Training base & 3,800 \\
& Test base & 504 \\
Architecture & $9-5-1$ Feed-forward MLP \\
Activation functions & Logsig-Logsig- linear \\
Training rule & Retropropagation error \\
Training MSE & $<0.0001$ \\
Iterations number & 3,000 \\
\hline
\end{tabular}

\subsection{Model test}

We designed a neural network-based model by taking into account the dependence on temperature and relative humidity at the measurement point, in addition to the dependence on gas nature when the sensor is placed in a dynamic environment. To illustrate this effect we change concentration, and then we note the variation of the sensor's resistance. Figure 5a shows the difference between the database and the ANN model for the sensor's sensitivity feature. Figure $5 \mathrm{c}$ shows the simulation error. The 
difference between the database and the ANN model for the dependence on temperature and relative humidity is shown in Figure 5 b.

Figure 5. (a) Model and database TGS822 sensor's sensitivity feature. (b) Model and database TGS822 dependence on temperature and relative humidity. (c) Simulation error vs. concentration.

(a)

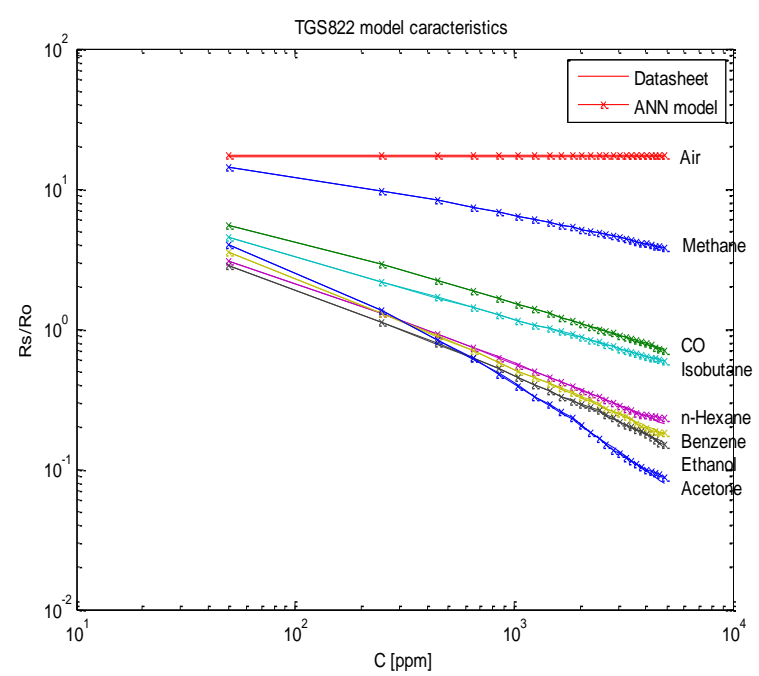

(b)

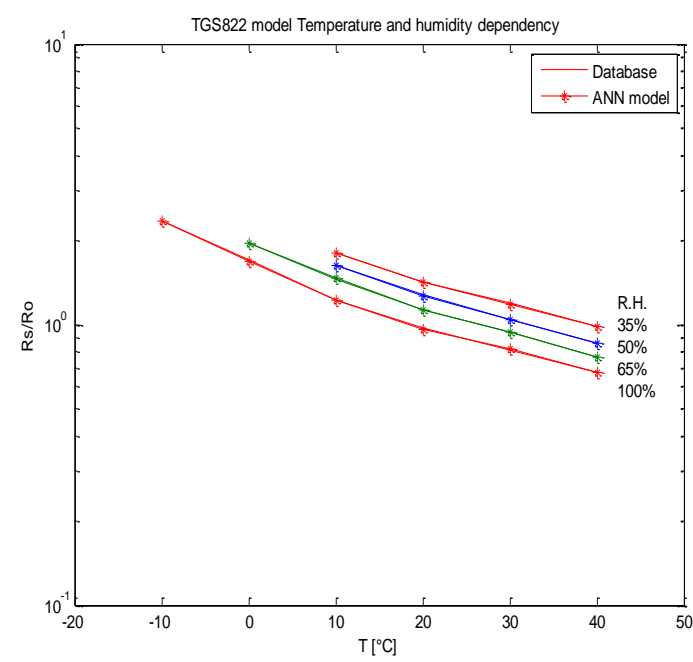

(c)

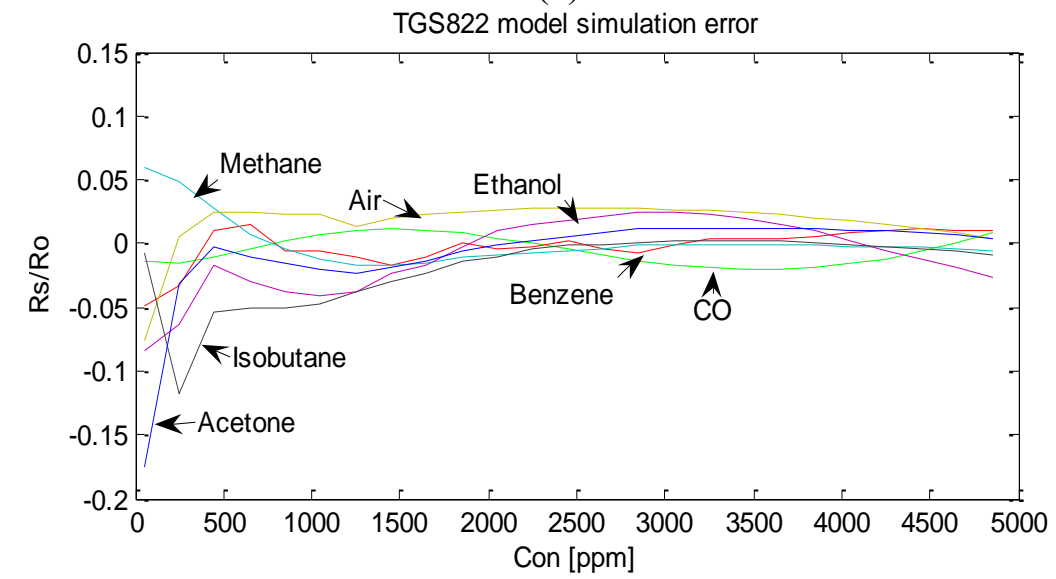

\subsection{Implementation of TGS822 model}

By using the ABM (Analog Behavioral Modeling) PSPICE Library components and the results (optimal architecture, bias and weights of the network) the sensor model designed previously is implemented as a component in the PSPICE simulator library.

In order to test the model introduced on PSPICE simulator, it has been implemented in the electrical circuit shown in Figure 6. 
Figure 6. Simulation circuit.

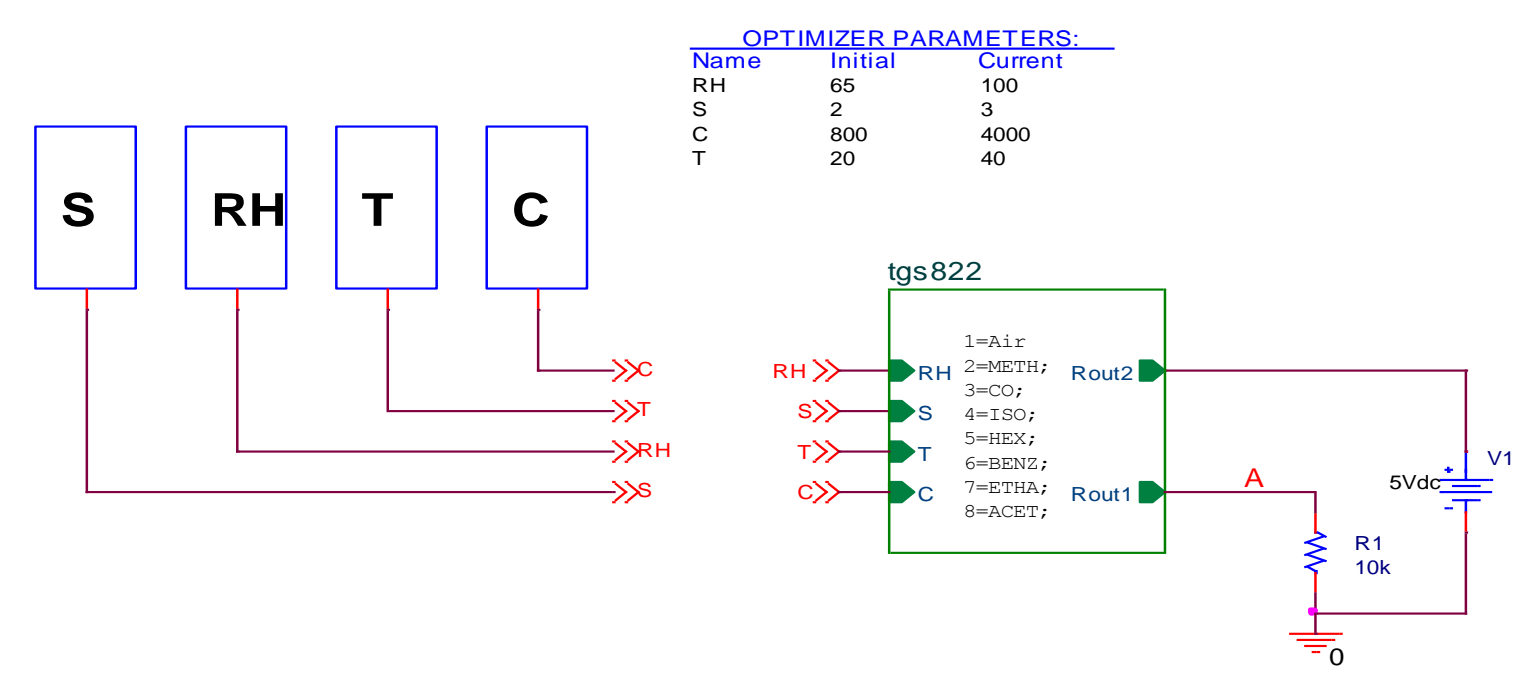

\subsection{Simulation results}

Temperature is fixed at $20 \mathrm{C}$, relative humidity at $65 \%$, concentration is varied from 300 to 5,000 ppm. A PARAMETRIC DC SWEEP analysis gives the variation of the résistance against the concentration $\mathrm{C}$ with different gas. Results are represented in Figure 7 where:

- $R s=\left(\frac{\mathrm{V} 1}{V(A)}-1\right) \mathrm{R} 1$

- $\mathrm{R} 1=\mathrm{Ro}=10 \mathrm{k} \Omega$

Figure 7. Variation of the résistance ratio (Rs/Ro) vs. gas concentration $\mathrm{C}$ at $20{ }^{\circ} \mathrm{C}$ and $65 \% \mathrm{RH}$.

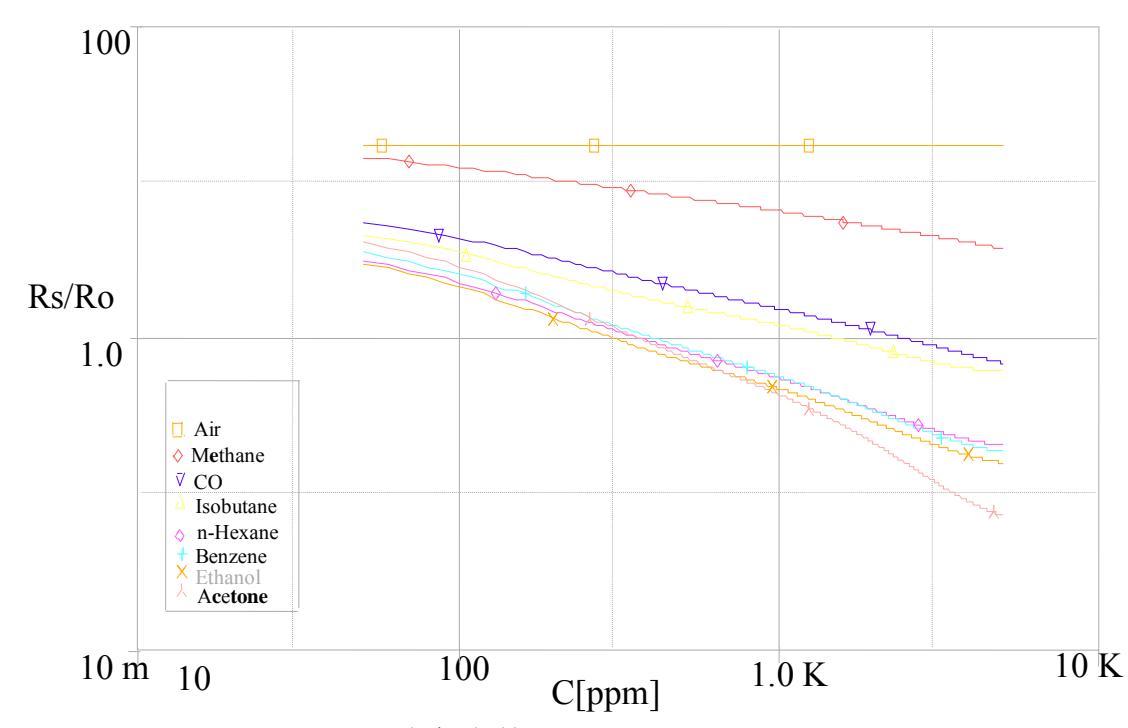

$(5 / \mathrm{V}(\mathrm{A}))-1$

By analogy to the first test, we carried out a second test. With a PARAMETRIC DC SWEEP analysis, we fixed the gas as ethanol, the concentration to $300 \mathrm{ppm}$ and the relative humidity RH as a parameter then we varied the temperature from -10 to $40 \mathrm{C}$. Figure 8 illustrates the obtained results. 
Figure 8. Variation of the résistance ratio (Rs/Ro) vs. temperature T at $300 \mathrm{ppm}$ of ethanol.

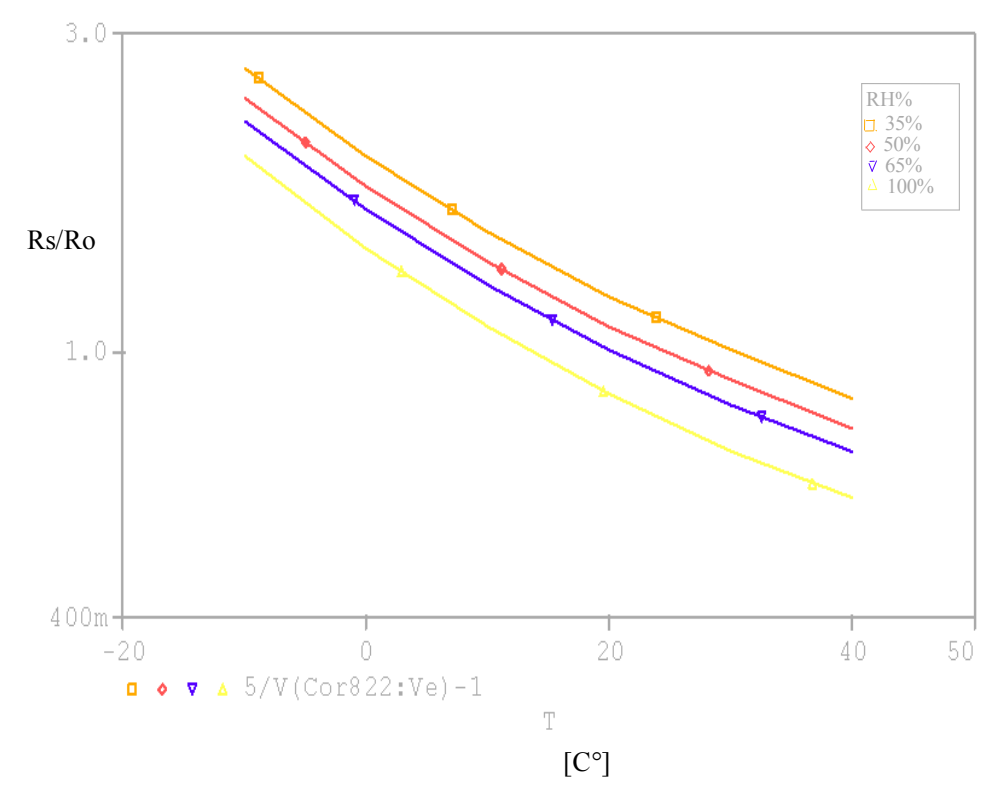

\section{Corrector}

Using the circuit in Figure 3 and by analogy to the model we design the corrector. The database is arranged as $\left(\mathrm{T}, \mathrm{RH}, \mathrm{V}_{\mathrm{RL}}, \mathrm{V}_{\mathrm{S}}\right)$ where:

T: Absolute temperature

RH: Relative humidity

$\mathrm{V}_{\mathrm{RL}}$ : Sensor's output voltage

Vs: Corrector's output voltage

The generation of the training base and test base is similar to that of the model's one. However, in the corrector, the temperature $\mathrm{T}$, relative humidity $\mathrm{RH}$ and the sensor's output voltage $\mathrm{V}_{\mathrm{RL}}$ are taken as inputs, and the corrector's output voltage Vs is taken as output. The diagram of Figure 9 illustrates the methodology used in the corrector design.

Figure 9. Modeling of the TGS822 corrector.

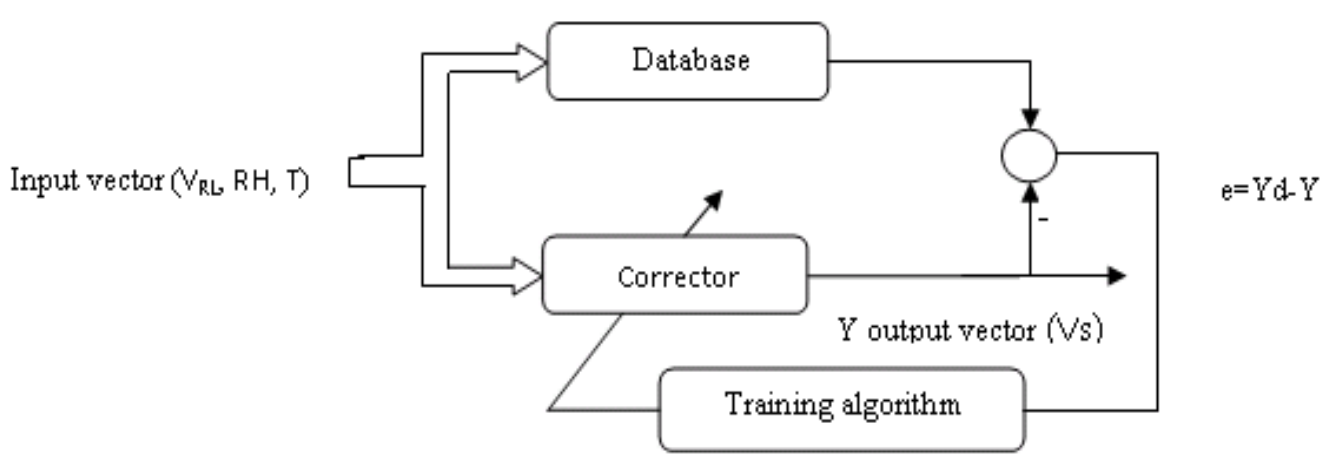

The corrector was trained in a similar manner as in the case of the model. After many tests, the optimized architecture which produces the smallest error is summarized in Table 2. 
Table 2. Summary of the corrector's optimized parameters.

\begin{tabular}{lll}
\hline Property & Characteristic & \\
\hline Database & Training base & 3,800 \\
& Test base & 504 \\
Architecture & $9-8-1$ Feed-forward MLP \\
Activation functions & Logsig-Logsig- linear \\
Training rule & Retropropagation error \\
Training MSE & $<0.00001$ \\
Iterations number & 5,000 \\
\hline
\end{tabular}

\subsection{Corrector test}

We designed an ANN-based corrector for the TGS822. To illustrate the effect of this corrector we change concentration, and then we note the variation of the corrector's output. Figure 10a shows that the corrector correctly linearizes the sensor's sensitivity feature (representation is in linear scale). The corrector's effect in environmental parameters dependency is shown in Figure 10b.

Figure 10. (a) TGS822 feature effect (b) TGS822 corrector temperature and relative humidity dependency effect (c) Corrector sensitivity simulation error.

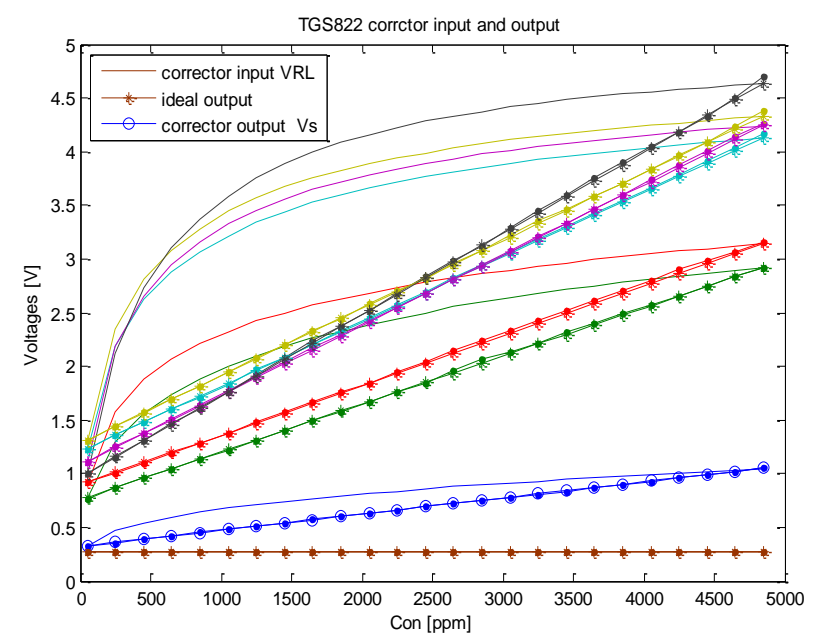

(a)



(b)

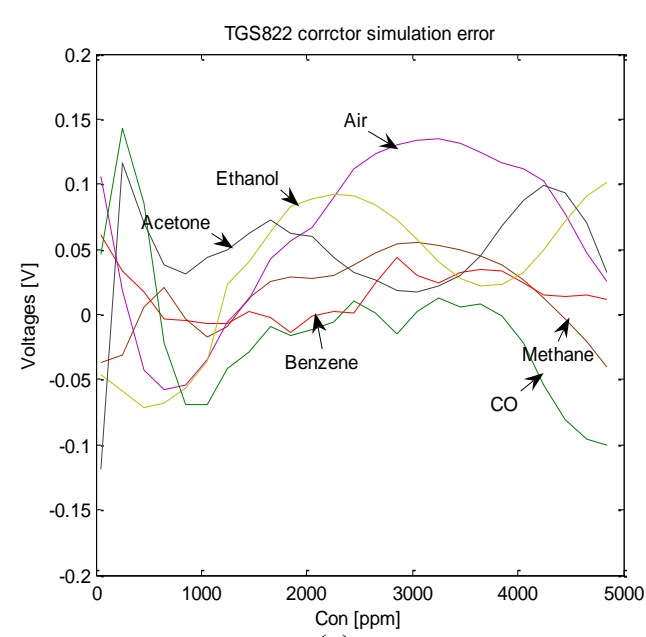

(c) 


\subsection{Implementation of the TGS822 corrector}

In order to test the effect of the corrector on electrical environment, the latter is implemented on the PSPICE simulator. The corrector and the TGS822 model have been implemented in the circuit shown in Figure 11.

Figure 11. Electrical circuit of the corrector test.

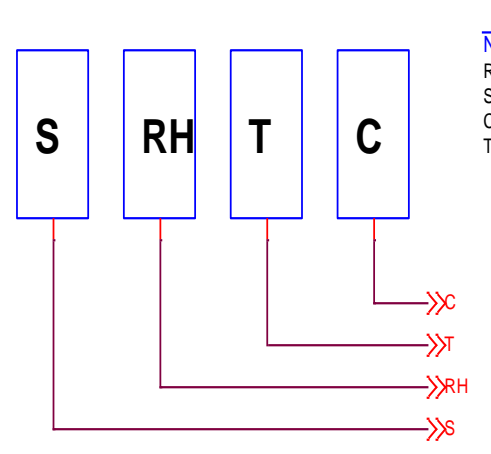

\begin{tabular}{lll}
\multicolumn{3}{c}{ OPTIMIZER PARAMETERS: } \\
\hline Name & Initial & Current \\
$\mathrm{RH}$ & 65 & 100 \\
$\mathrm{~S}$ & 2 & 3 \\
$\mathrm{C}$ & 800 & 4000 \\
$\mathrm{~T}$ & 20 & 40
\end{tabular}

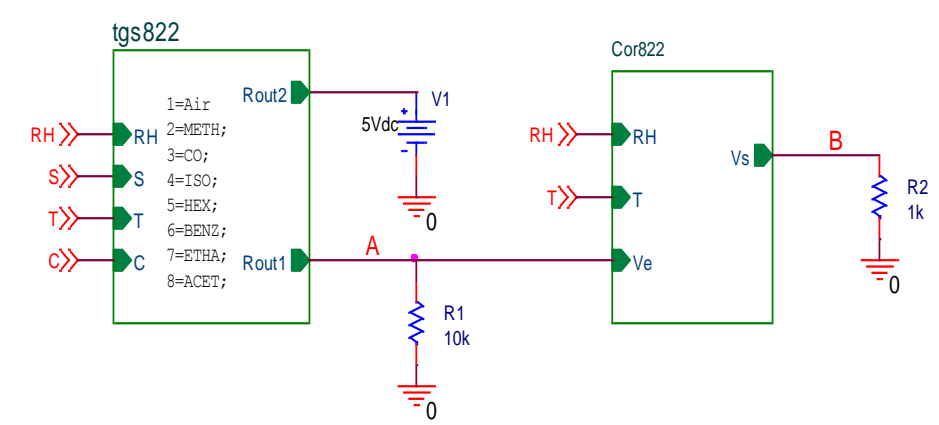

\subsection{Simulation results}

Temperature is fixed at $20{ }^{\circ} \mathrm{C}$, relative humidity at $65 \%$ and concentration is varied from 300 to $5,000 \mathrm{ppm}$. A PARAMETRIC DC SWEEP analysis gives the results represented in Figure 12 with different gases.

Figure 12. Variation of the corrector's input and output vs. concentration $\mathrm{C}$ at $20{ }^{\circ} \mathrm{C}$ and $65 \% \mathrm{RH}$.

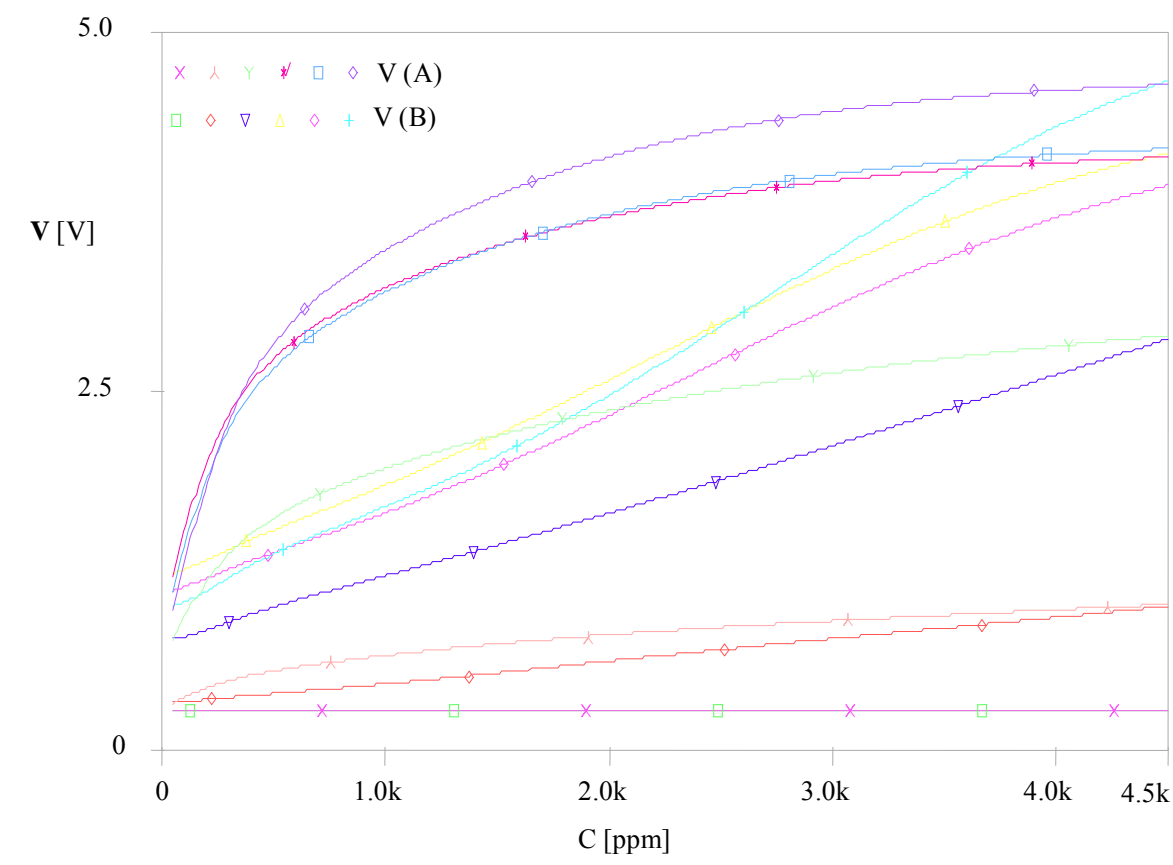


The parametric simulation shows that the corrector linearizes the sensor's response whatever the gas. By analogy to the first test, we carried out a second test with a PARAMETRIC DC SWEEP analysis, we fixed the gas as ethanol, the concentration to $300 \mathrm{ppm}$ and the relative humidity $\mathrm{RH}$ as a parameter then we varied the temperature from -10 to $40^{\circ} \mathrm{C}$. Figure 13 illustrates the obtained results.

Figure 13. Variation of the corrector's input and output vs. temperature $T$ at $300 \mathrm{ppm}$ of ethanol.

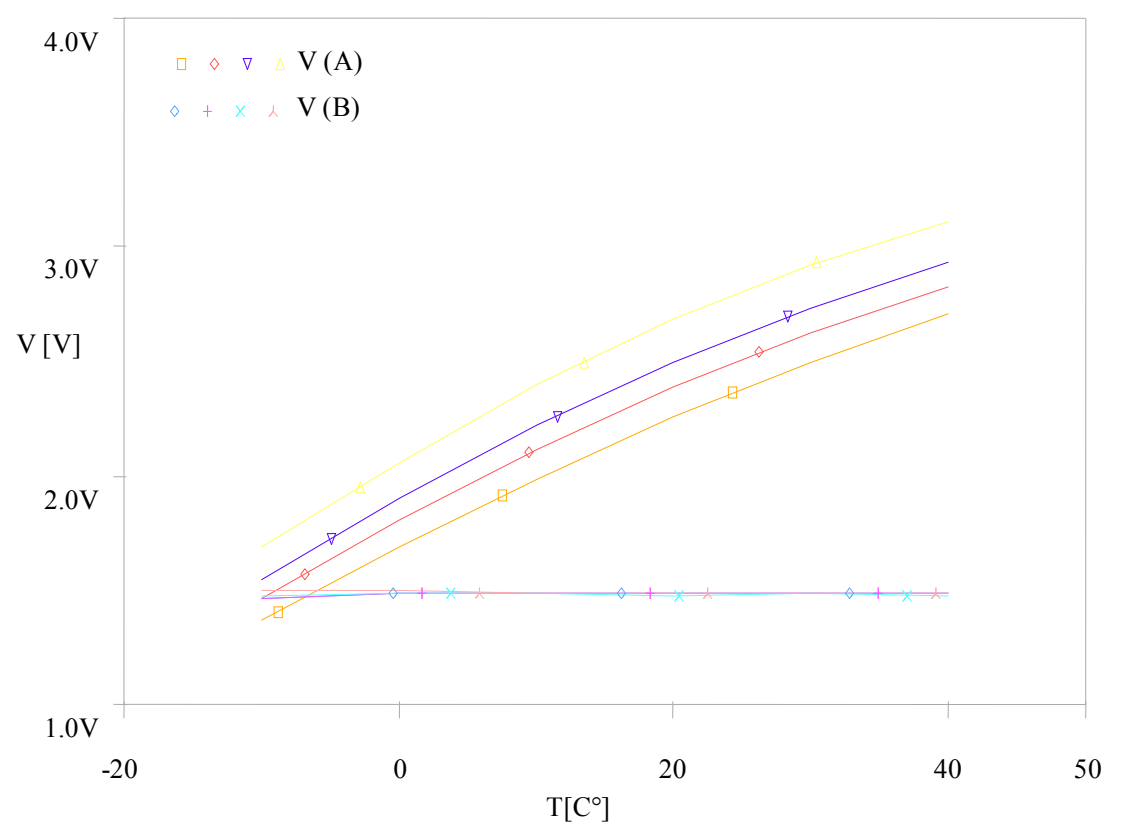

The response of the parametric simulation shows that the corrector eliminates the environmental parameters' effect.

\section{The Selective Module}

By analogy to the model and the corrector we design the selective module. The database is arranged as (Ve1, Ve2, Ve3, Vsn, Vsc), where Ve1, Ve2, Ve3 are taken as input of the selective model and they are the output of the correctors, Vsn is taken as output and it defines the gas nature, Vsc is taken as output and it defines the gas concentration. Note that:

- Vcon is a voltage varing linearly from 0.4 to $5 \mathrm{~V}$ corresponding to a concentration variation from 400 to 5,000 ppm.

- Vsc is a voltage varing from $1 \mathrm{~V}$ to $10 \mathrm{~V}$, the table 3 indicates their matched gases.

Table 3. Voltages and their matched gases.

\begin{tabular}{cc}
\hline Gas & Voltage [V] \\
\hline Air & 1 \\
Methane & 2 \\
CO & 3 \\
\hline
\end{tabular}


Table 3. Cont.

\begin{tabular}{cc}
\hline Isobutane & 4 \\
Ethanol & 5 \\
Benzene & 6 \\
$n$-Hexane & 7 \\
Acetone & 8 \\
Hydrogen & 9 \\
Propane & 10 \\
\hline
\end{tabular}

The selective module was trained in a similar manner as in the case of direct model and corrector. The optimized architecture characteristics are summarized in Table 4.

Table 4. Summarizing of the optimized parameters of selective module.

\begin{tabular}{llc}
\hline Property & Characteristic & \\
\hline Database & Training base & 250 \\
& Test base & 50 \\
Architecture & $5-2-2$ Feed-forward MLP & \\
Activation functions & Logsig-Logsig- linear & \\
Training rule & Retropropagation error \\
Training MSE & $<10^{-6}$ \\
Iterations number & 1,000 \\
\hline
\end{tabular}

\subsection{Implementation of the selective module}

The selective module was implemented in the same manner as the sensor model and the corrector. The schematic shown in Figure 14 represents the electrical circuit used for testing the technique used in electrical environment.

Figure 14. Electrical circuit of the method test.



\subsection{Simulation results}

\subsubsection{Concentration effect}

To study the concentration effect we fixed the temperature at $20 \mathcal{C}$, relative humidity at $5 \%$ and concentration is varied from 300 to $5,000 \mathrm{ppm}$. A DC SWEEP analysis gives the results represented in Figure 15 for the choice of the methane and Figure 16 for the choice of the CO, where $\mathrm{Ve} 1, \mathrm{Ve} 2$ and $\mathrm{Ve} 3$ are the corrector output, Vsng is the selective module output for the gas nature and Vsc is the selective module output for the gas concentration.

Figure 15. Variation of the inputs and the outputs the selective module vs. concentration $\mathrm{C}$ at $20{ }^{\circ} \mathrm{C}$ and $65 \% \mathrm{RH}$ for the detection of (a) methane (b) $\mathrm{CO}$.

(a)

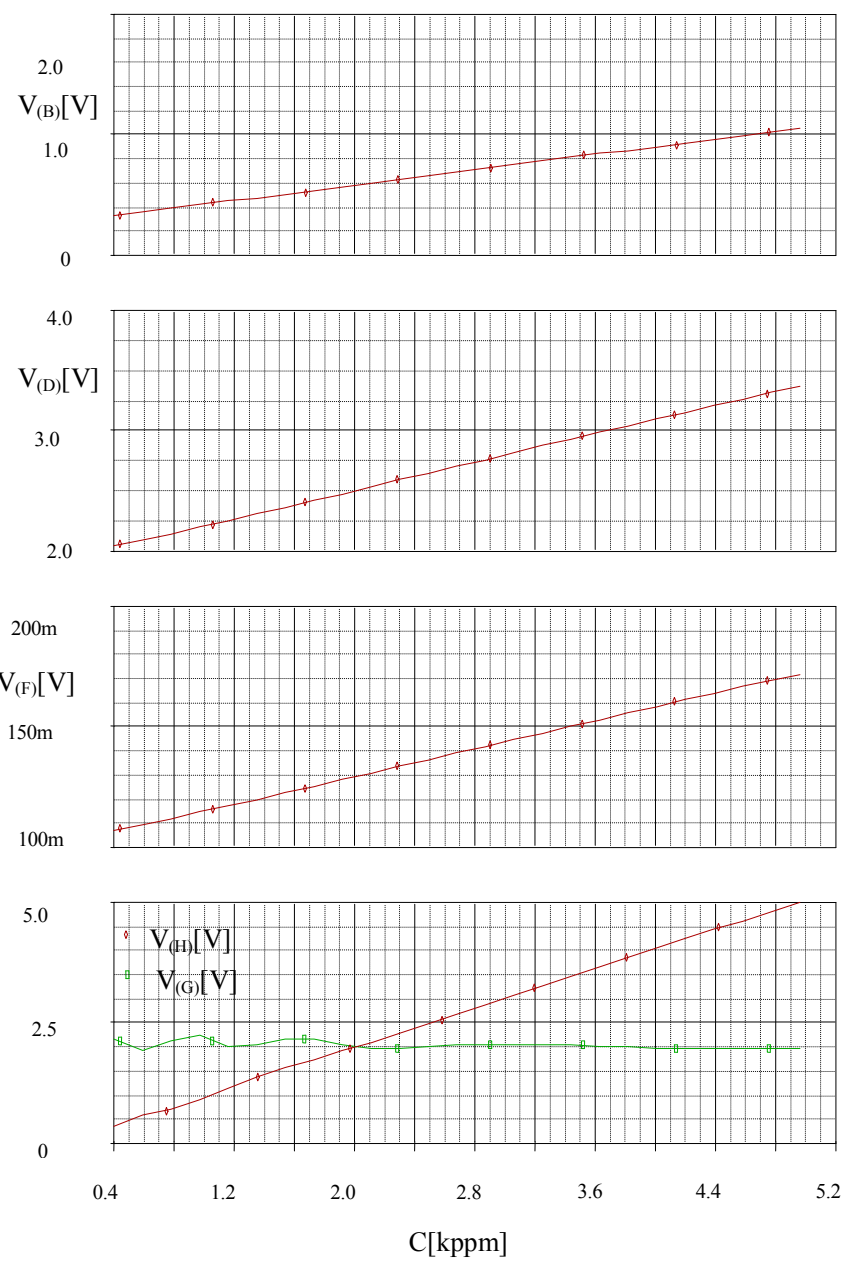

(b)


0

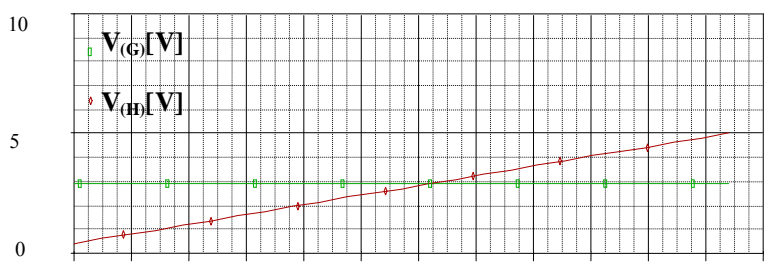

0.4

$4.4 \quad 5.2$ 2.8
$\mathrm{C}[\mathrm{kppm}]$

Figure 15a shows that the method identifies the gas as methane $\left(\mathbf{V}_{(\mathbf{G})}=2 \mathrm{~V}\right.$, Table 3$)$, and the quantity of this gas (linear variation of $\mathbf{V}_{(\mathbf{H})}$ against $\mathrm{C}$ ). Figure $15 \mathrm{~b}$ shows that the method discriminates the gas as $\mathrm{CO}\left(\mathbf{V}_{(\mathbf{G})}=3 \mathrm{~V}\right.$, Table 3$)$, and the quantity of this gas (linear variation of $\mathbf{V}_{(\mathbf{H})}$ against $\mathrm{C}$ ). 


\subsubsection{Temperature effect}

The effect of the temperature is analyzed with a DC SWEEP analysis. 2,500 ppm of hydrogen is used for the test. We fixed the relative humidity to $65 \%$ then we varied the temperature from -10 to $40 \mathrm{C}$. Figure 16 illustrates the results of this simulation.

Figure 16. Variation of the corrector and the selective module's inputs and outputs vs. the temperature $\mathrm{T}$ at $65 \% \mathrm{RH}$ for $2,500 \mathrm{ppm}$ of hydrogen.
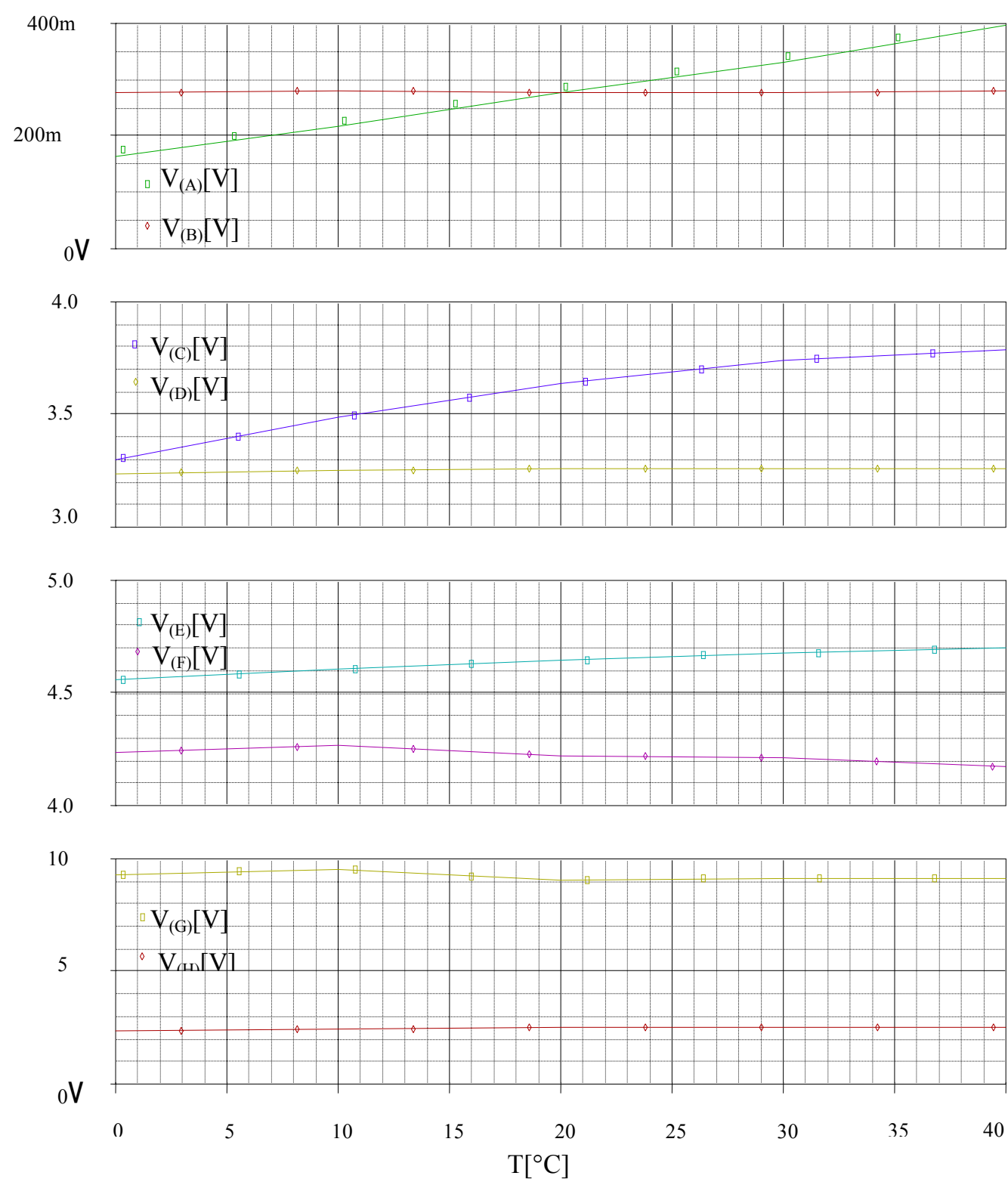

Figure 16 shows that the method discriminates $2,500 \mathrm{ppm}$ of hydrogen $\left(\mathbf{V}_{(\mathbf{H})}=2.5 \mathrm{~V}, \mathbf{V}_{(\mathbf{G})}=9 \mathrm{~V}\right)$, and stay unchanged vs. the temperature. 


\subsubsection{Relative humidity effect}

We introduce the humidity effect with a DC SWEEP analysis. Figure 17 illustrates the relative humidity influence on the corrector and the selective module outputs.

Figure 17. Variation of the inputs and the outputs of the correctors and the selective module vs. the relative humidity $\mathrm{RH}$ at $20{ }^{\circ} \mathrm{C}$ for the detection of $1,300 \mathrm{ppm}$ of benzene.

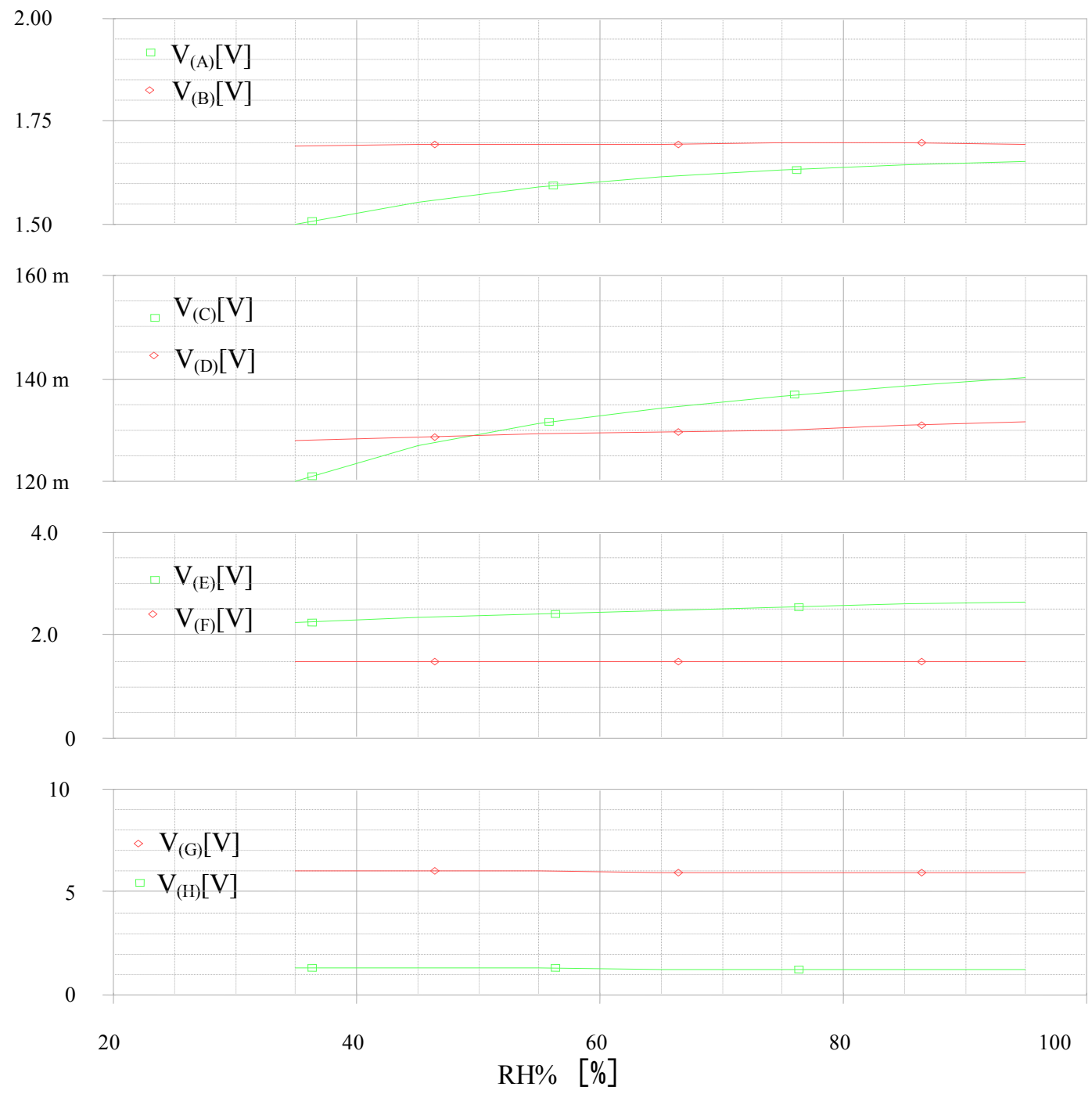

Figure 17 shows that the method discriminates $1,300 \mathrm{ppm}$ of benzene $\left(\mathbf{V}_{(\mathbf{H})}=1.3 \mathrm{~V}, \mathbf{V}_{(\mathbf{G})}=6 \mathrm{~V}\right)$, and stay unchanging vs. the humidity.

\section{Conclusions}

In this paper we modeled three TGS8xx sensors using an artificial neural network sensor. It accurately reproduces the behavior of the gas sensors in a dynamic environment by taking into account the nonlinearity of their responses, the dependence on temperature and relative humidity at the measurement point, in addition to the dependence on the gas nature. The proposed ANN models were tested and implemented as components of the PSPICE simulator library. We used the ANN capability 
to correct the nonlinear response and to compensate gor temperature and relative humidity influences on the target sensor variations which is tested by Matlab and PSPICE simulation results. We wire all the components with the selective module with the aim of increasing the selectivity. The new technique discriminates and qualitatively and quantitatively measured seven gase,s tested between 300 and 5,000 ppm, with few sensors and this method can be easily extended to other kinds of sensors.

\section{References and Notes}

1. Leonard, M. Neural Network Applications in Device and Subcircuit Modeling for Circuit Simulation. Thesis, Philips Research Laboratories: Eindhoven, the Netherlands, 2003.

2. Patra, J.C.; Van den Bos, A.; Kot, A.C. An ANN-based smart capacitive pressure sensor in dynamic environment. Sens. Actuat. 2000, 86, 26-38.

3. Jung, Y.K.; Sung, W.K.; Tae, Z.S.; Myung, K.Y.; Kyu, S.L. Design of a smart gas sensor system for room air- cleaner of automobile -thick-film metal oxide semiconductor gas sensor. IEEE 2006, 20, 72-75.

4. Kieu, A.N. Etude d'un systeme multicapteur pour la detection selective des gaz. Thèse de doctorat. Paul Cezanne University: Aix-Marseille III, France, 2006.

5. Elena, G.; Robert, M.N. Smart intelligent and cogent microsensors intelligence for sensors and sensors for intelligence. NSTI Nanotechnol. 2004, 1, 443-446.

6. Serge, Z. Gas sensor applications of oxygen-ionic electrolytes development of their electron model. Sens. Actuat. B 2007, 130, 488-496.

7. Andrei, P.; Fields, L.L.; Zheng, J.P.; Cheng, Y.; Xiong, P. Modeling and simulation of single nanobelt $\mathrm{SnO} 2$ gas sensors with FET structure. Sens. Actuat. 2007, 128, 226-234.

8. Fort, A.; Rocchi, S.; Santos, S.; Spinicci, R.; Vignoli, V. Electronic noses based on metal oxide gas sensors the problem of selectivity enhancement. In Proceedings of the IEEE Instrumentation and Measurement Technology Conference, Como, Italy, May18-20, 2004; pp. 599-604.

9. Bendahan, M.; Guerin, J.; Boulmani, R.; Aguir, K. $\mathrm{WO}_{3}$ sensor response according to operating temperature: Experiment and modeling. Sens. Actuat. 2007, 124, 24-29.

10. Iglesias, G.E.; Iglesias, E.A. Linearization of transducer signal using an analog to digital converter. IEEE Trans. Instrum. Meas. 1988, 37, 53-57.

11. Vargha, B.; Zoltán, I. Calibration algorithm for current-output R-2R ladders. IEEE Trans. Instrum. Meas. 2001, 50, 1216-1220.

12. Kaliyugavaradan, S.; Sankaran, P.; Murti, V.G.K. A new compensation scheme for thermistors and its implementation for response linearization over a wide temperature range. Sensors 2007, 7, $15-27$.

13. Cristaldi, L.; Ferro, A.; Lazzaroni, M.; Ottoboni, R. A linearization method for comercial hall- effect current transducer. IEEE Trans. Instrum. Meas. 2001, 50, 1149-1153.

14. James, H.T.; Antoniotti, A.J. Linearisation algorithm for computer aided control engineering. IEEE Contr. Syst. 1993, 13, 58-64.

15. Patranbis, D.; Gosh, D. A novell software based transducer linearizer. IEEE Trans. Instrum. Meas. 1989, 38, 1122-1126. 
16. Malcovati, P.; Leme, C.A.; O'Leary, P.; Maloberti, F.; Baltes, H. Smart sensor interface with A/D conversion and programmable calibration. IEEE J. Solid-State Circuits 1994, 29, 963-966.

17. Bin, G.; Bermak, A.; Philip, C.H.C.; Guizhen, Y. A monolithic integrated $4 \times 4$ tin oxide gas sensor array with on-chip multiplexing and differential readout circuits. Solid State Electron. 2007, $51,47-54$.

18. Ivanov, P.; Llobet, E.; Blanco, F.; Vergara, A.; Brezmes, J.; Vilanova, X.; Gracia, I.; Cané, C.; Correig, $\mathrm{X}$. On the effects of the materials and the noble metal doping to NO2 detection. Sens. Actuat. B 2006, 118, 311-317.

19. Flitti, F.; Guo, B.; Far, A.; Bermak, A. A new gas recognition technique using on chip sensor array measurements. In Proceedings of the 14th IEEE International Conference on Electronics, Circuits and Systems, Marrakech, Morocco, December 11-14, 2007; pp. 50-53.

20. Ding, H.; Ge, H.; Liu, J. High performance of gas identification by wavelet transform based fast feature extraction from temperature modulated semiconductor gas sensor. Sens. Actuat. B 2005, 107, 749-755.

21. Vergara, A.; Llobet, E.; Brezmes, J.; Ivanov, P.; Vilanova, X.; Gràcia, I.; Cané, C.; Correig, X. Optimised temperature modulation of metal oxide micro-hotplates gas sensors through multi-level pseudo random sequences. Sens. Actuat. B 2005, 111-112, 271-280.

22. Ionescu, R.; Hoel, A.; Granqvist, C.G.; Llobet, E.; Heszler, P. Low level detection of ethanol and $\mathrm{H} 2 \mathrm{~S}$ with temperature modulated $\mathrm{WO}_{3}$ nanoparticle gas sensors. Sens. Actuat. B 2005, 104, $132-139$.

23. Figaro Gas Sensor Company. Technical Information on Usage of TGS Sensors for Toxic and Explosive Gas Leak Detectors; Figaro Gas Sensor Company: Osaka, Japan, 2000.

(C) 2009 by the authors; licensee Molecular Diversity Preservation International, Basel, Switzerland. This article is an open-access article distributed under the terms and conditions of the Creative Commons Attribution license (http://creativecommons.org/licenses/by/3.0/). 